\title{
EVALUATING SOUTH AFRICAN CHENIN BLANC WINE STYLES USING AN LC-MS SCREENING METHOD
}

\section{ASTRID BUICA ${ }^{\mathrm{a}^{*}}$, JEANNE BRAND ${ }^{\mathrm{a}}$, CHRISTINE WILSON ${ }^{\mathrm{a}}$, MARIETJIE STANDER ${ }^{\mathrm{b}}$}

\begin{abstract}
Sensory evaluation is the approach currently used when evaluating the style of a South African Chenin Blanc wine. Using an untargeted LC-HRMS approach, a number of wine samples previously attributed to the three recognized styles were used to build a statistical model which was further used to predict to which style group additional samples belonged. This application can be considered proof of principle.
\end{abstract}

Keywords: Chenin Blanc wine styles; LC-MS; screening; sorting

\section{INTRODUCTION}

With the help of chemometrics, large amounts of data generated for wine analyses can be used for statistical modelling. Classification and discrimination of samples, quantification of certain classes of compounds, and prediction was successfully achieved in wine research using chemometrics [1-4]. Sensory evaluation is also a field that makes use of statistics, in experimental design, panel performance testing, and, of course, data handling

\footnotetext{
a Department of Viticulture and Oenology, Stellenbosch University, Private Bag X1, Matieland 7602, South Africa

b Mass Spectrometry Unit, Central Analytical Facility, Stellenbosch University, Private Bag X1, Matieland 7602, South Africa

*Corresponding author: abuica@sun.ac.za
} 
[5-7]. Various sensory methods have been developed hand in hand with their own data handling approach [8]. The results are generally used for discrimination and classification between samples, but not for prediction. The question is - with the appropriate analysis technique, can sensory behaviour be predicted?

Chemical analysis and sensory evaluation are the two means through which wines are assessed, but linking the two has usually met with limited success. This is maybe counterintuitive, since the chemical composition will dictate the flavour, taste, and mouth-feel of a wine through the various compounds present in the sample.

The issue resides rather in the approach. Sensory evaluation has a holistic and comprehensive approach, in the sense that the entire product is evaluated at once and it is seen as a whole and not the sum of parts. However, looking into the chemical composition of a wine using targeted separation techniques reduces the whole to the sum of parts. In that case, sensory and chemistry results seldom correlate well. On the other hand, an untargeted approach has more chances of succeeding. Some analytical techniques, such as UV/Vis and IR spectroscopy, have been utilised successfully in the classification, discrimination, and quantification of certain classes of compounds present in wine [4,9-11]. Similarly, untargeted or comprehensive chromatographic approaches coupled with chemometrics have a better chance of succeeding because they are information-rich. The use of untargeted analytical techniques to profile, model, and predict sensory behaviour is an expanding area of research. The applications are at the moment relatively few and some are heavily chemistry-based while the sensory aspects are more informal [12-15].

Chenin Blanc is one of the most important white wine cultivars in South Africa. It has received a lot of attention and accolades in the past years and more research than ever is dedicated to this versatile cultivar. According to the Chenin Blanc Association of South Africa, there are three recognized dry wine styles, Fresh and Fruity (FF), Rich and Ripe Unwooded (RRU), and Rich and Ripe Wooded (RRW) [16]. They are traditionally established with the aid of expert sensory evaluation, but the cost and the (subjective) human factor are aspects to be taken into account. Also, the number of samples that can be judged in one tasting session is limited [17].

A more objective and robust way of assessing and attributing these styles can be the use of chemical analysis. Chemical composition can be a better way for discriminating between style groups using an untargeted approach such as LC-MS. This approach is information-rich and offers numerous possibilities for statistical data modelling (PCA, PLS, HCA, etc.). Creating 
prediction models to include additional samples possibly without the need for sensory evaluation is a powerful tool for future applications. As always, there are some possible drawbacks to this approach, too, such as the need for data pre-processing that doesn't remove relevant information but helps build strong and reliable models. Additionally, creating a reliable model depends on the choice of training set (done by sensory evaluation) so the choice of appropriate (representative) samples for the training set still falls on the sensory assessment.

In this work, a sample set representative of the three Chenin Blanc wine styles was evaluated sensorially by a panel of expert judges. These wines and additional samples were analysed by LC-HRMS. The data obtained was used to create a statistical model used to predict to which of the styles the additional samples belonged, thus avoiding the need for sensorial evaluation for the additional samples.

\section{RESULTS AND DISCUSSION}

The sensory and chemistry data sets were treated separately and groupings of samples around the predefined styles were found for both sets.

\section{Sensory evaluation}

Sample repeats are close to each other, indicating good repeatability of the expert panel. Similar to previous findings, the sensory evaluation of Chenin Blanc wines leads to the formation of 2 rather than 3 defined groups. At first glance, RRW group is set apart from the FF and RRU wines which form a continuum rather than two distinct groups (Fig 1). Even though there is a trend for the FF-RRU wines to have FF wines on one side and RRU wine to the other, they do not make distinct groups. This is illustrated by the two wines that have been place with equal frequency in the FF and RRU groups, LKAP and WGHBV.

Moreover, looking at the dendrogram representation of the results, it becomes apparent that there are in fact four groups, and not only two (Fig 2). The dendrogram (Fig 2) shows that the grouping is not as clear as suggested by the configuration plot (Fig 1). The two samples that were placed by the judges equally in the FF and RRU groups, LKAP and WGHBV, are indeed placed with two other samples that belong to those groups, SVP to FF and BCG to RRU, respectively. On the other hand, one of the RRW samples, WGHV, is positioned with two samples that belong in the RRU group, REM and MP. This is not unusual, as often the wines described as rich and ripe unwooded are the most challenging to ascribe to a well-defined group. 
ASTRID BUICA, JEANNE BRAND, CHRISTINE WILSON, MARIETJIE STANDER

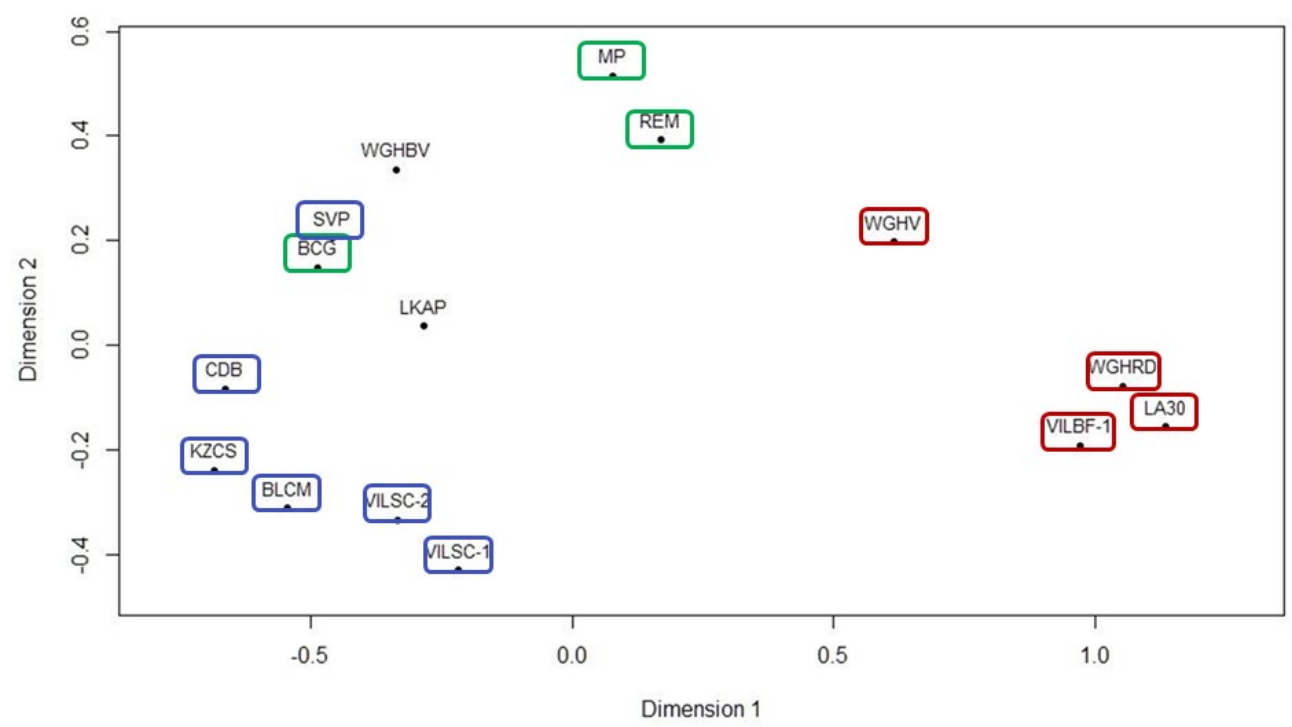

Figure 1. Configuration plot after directed sorting task. The styles are attributed as FF (blue), RRU (green), and RRW (red). The codes without frames are from samples that were attributed to two groups equally ( $F F$ and $R R U)$.

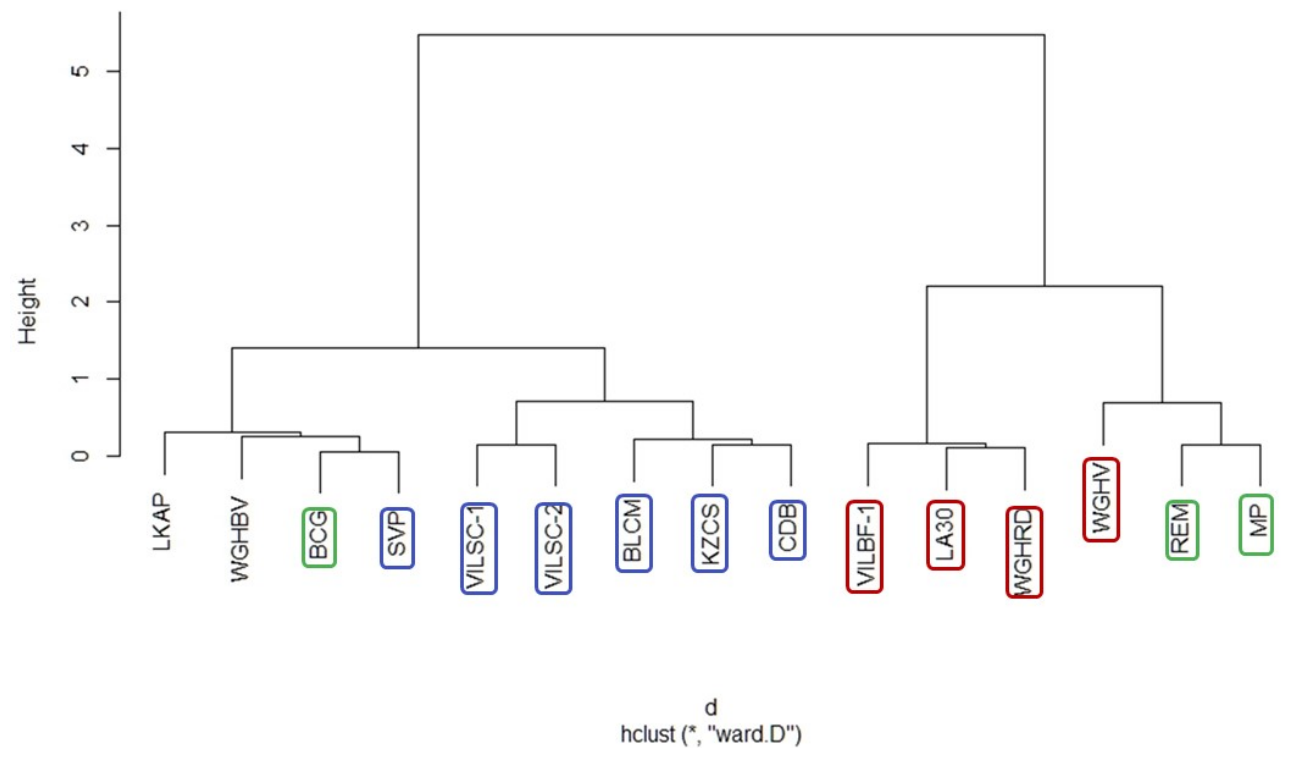

Figure 2. Dendrogram for the directed sorting task results. The styles are attributed as FF (blue), RRU (green), and RRW (red). The codes without frames are from samples that were attributed to two groups equally (FF and RRU). 


\section{LC-MS analysis}

The representation of all data, combining the results for positive and negative ionization modes, is shown in Fig $3 \mathrm{a}$ and $3 \mathrm{~b}$. The PCA model for all samples, including the wines not classified during the sensory evaluation, shows some grouping that is more obvious in the representation of PC1 and 3 (Fig $3 b$ ). In that case, the separation of wines from the RRW group seems more apparent than for PC1 and 2 (Fig 3a). The configuration is similar for chemistry and sensory data (Fig 1 vs Fig $3 b$ ) with a continuum between the FF and RRU groups and in opposite quadrants from RRW. The additional 'no class' samples are more difficult to ascribe to a style group when inspecting the PCAs.

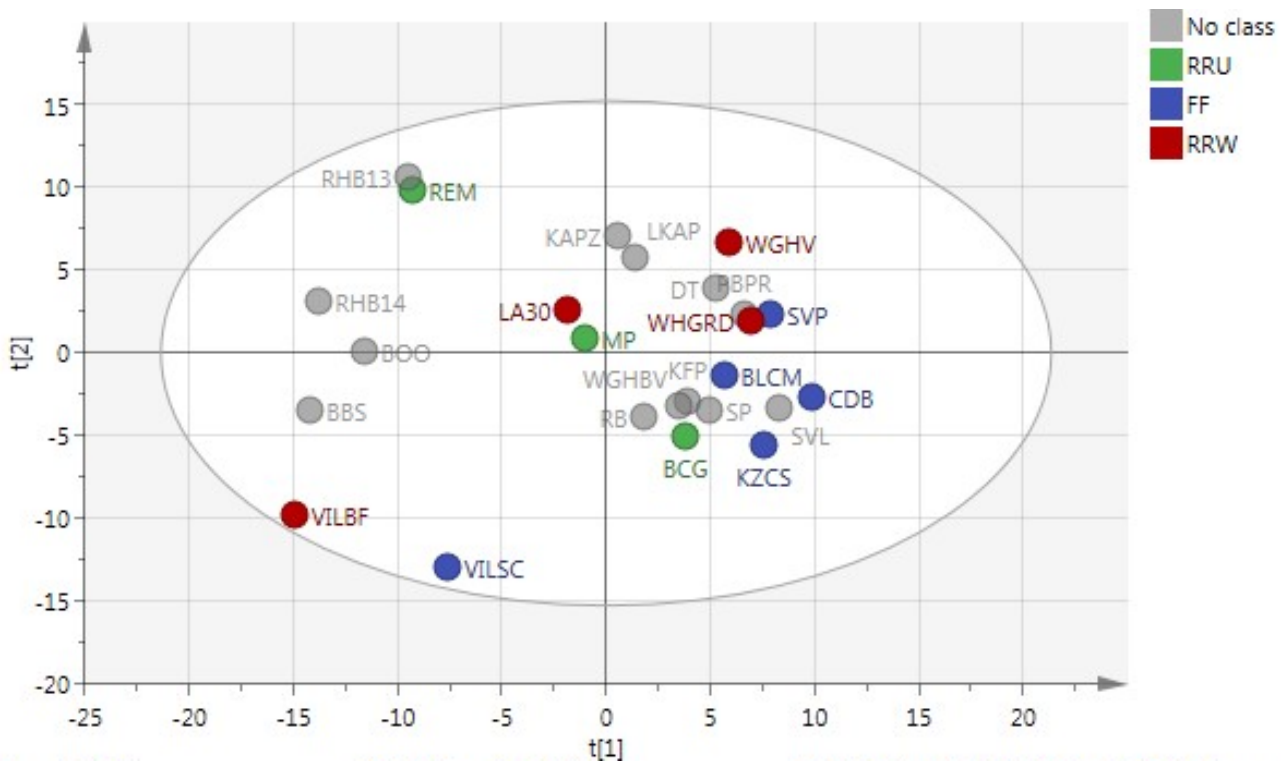

Figure 3a. Component 1 and 2 of the PCA-X model for the combined LC-HRMS results, no sample pre-processing $(n=25)$. Styles attributed by the sensory task.

'No class' samples were not included in the sensory task. 


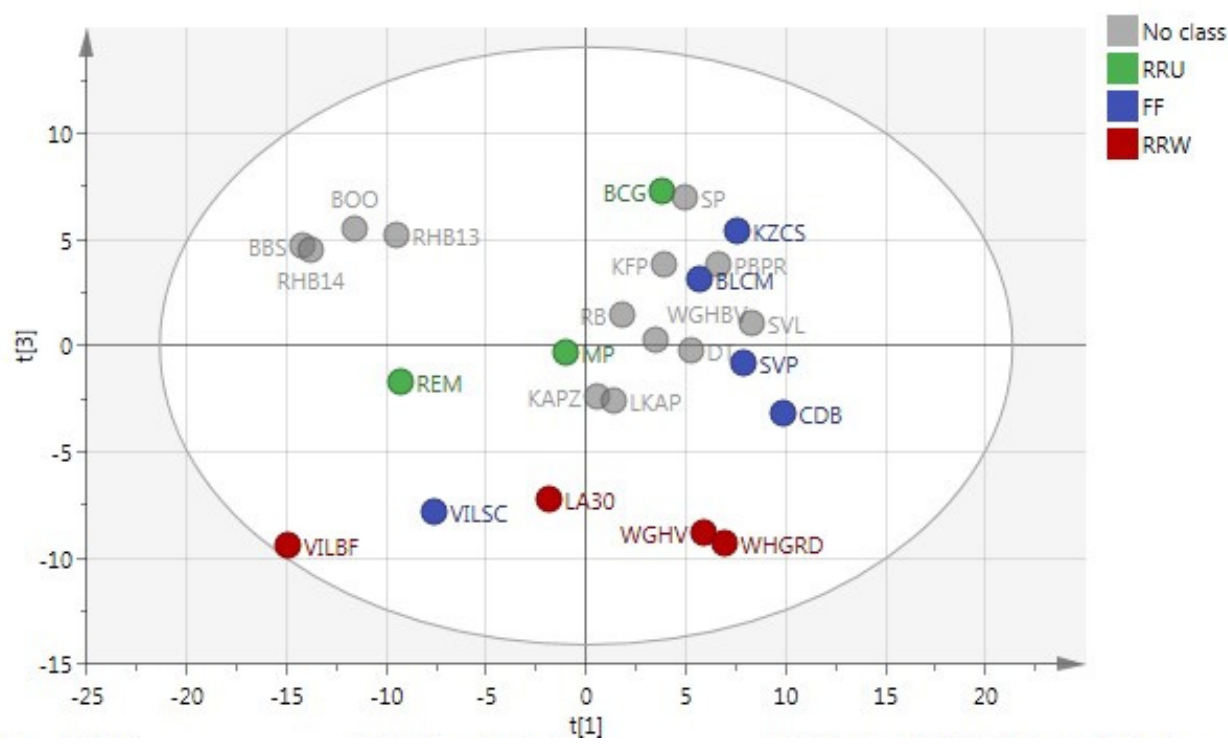

Figure 3b. Component 1 and 3 of the PCA-X model for the combined LC-HRMS results, no sample pre-processing $(n=25)$. Styles attributed by the sensory task. 'No class' samples were not included in the sensory task.

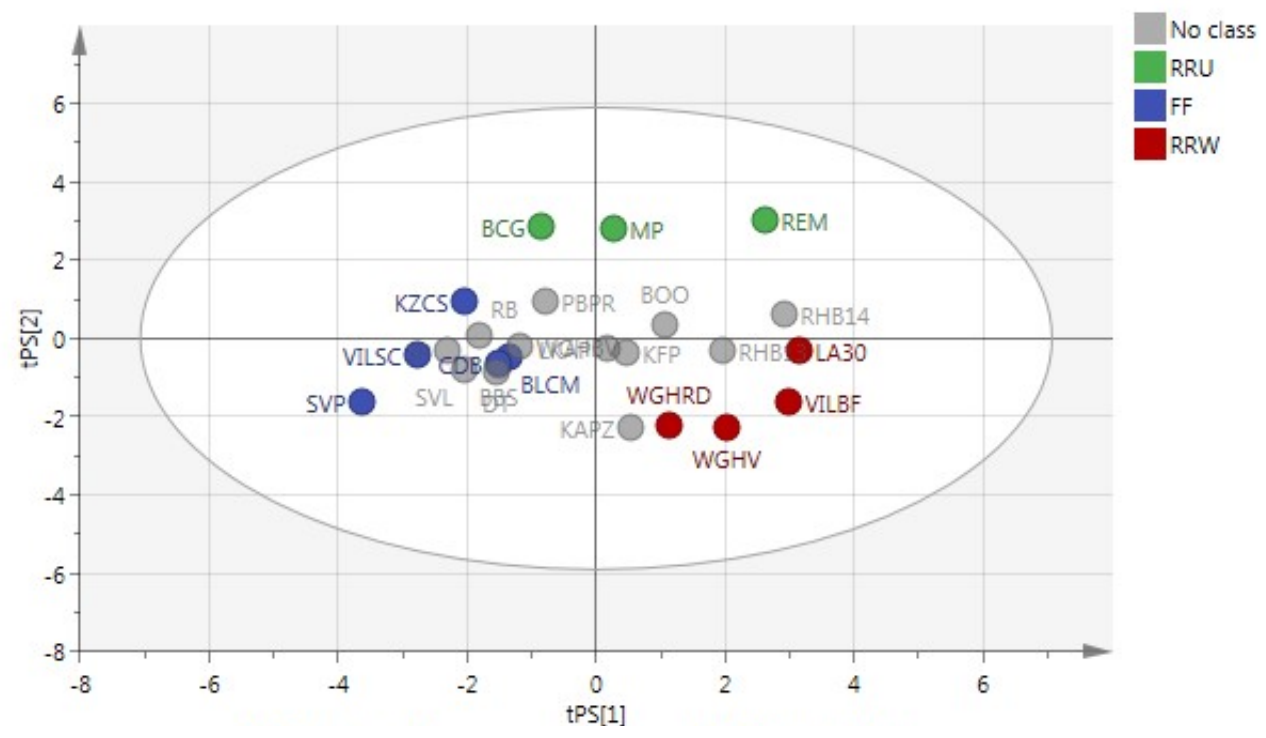

Figure 4a. PLS-DA model for positive ionization LCHRMS results, no sample pre-processing $(n=25)$. Styles attributed by the sensory task.

'No class' samples constitute the prediction set. 


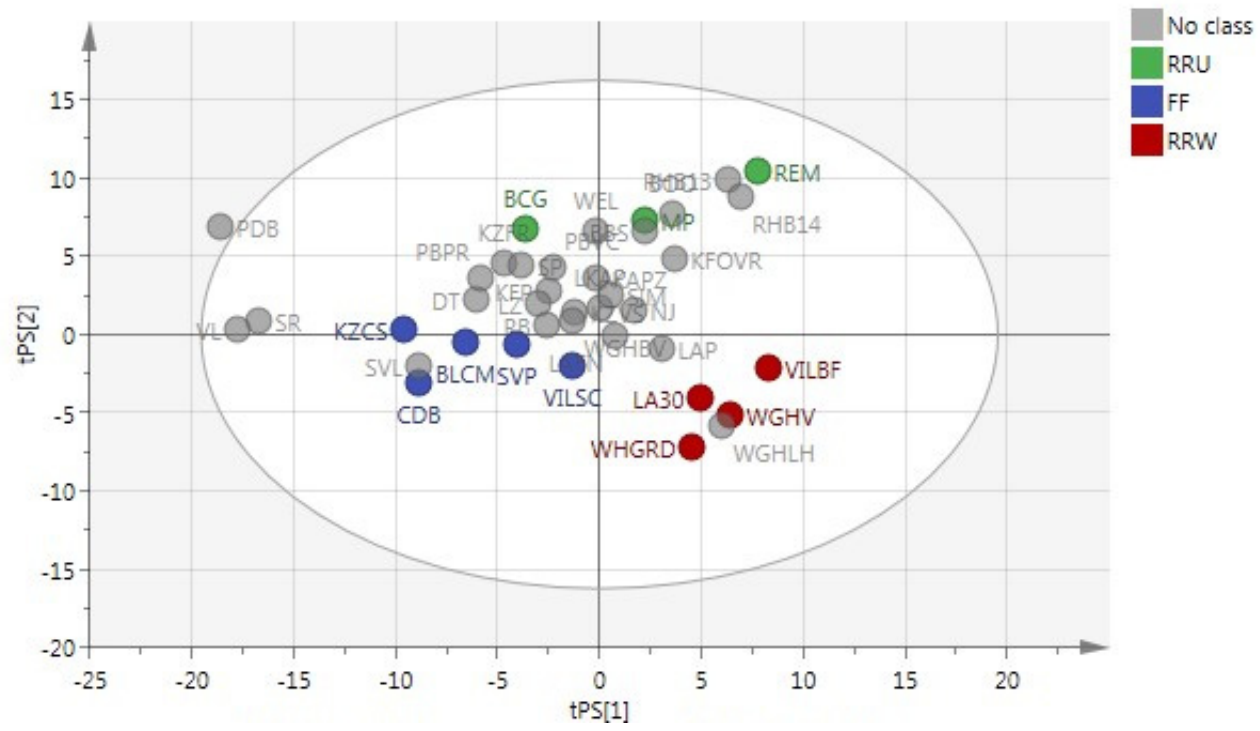

Figure 4b. PLS-DA model for negative ionization LCHRMS results, no sample pre-processing $(n=39)$. Styles attributed by the sensory task.

'No class' samples constitute the prediction set.

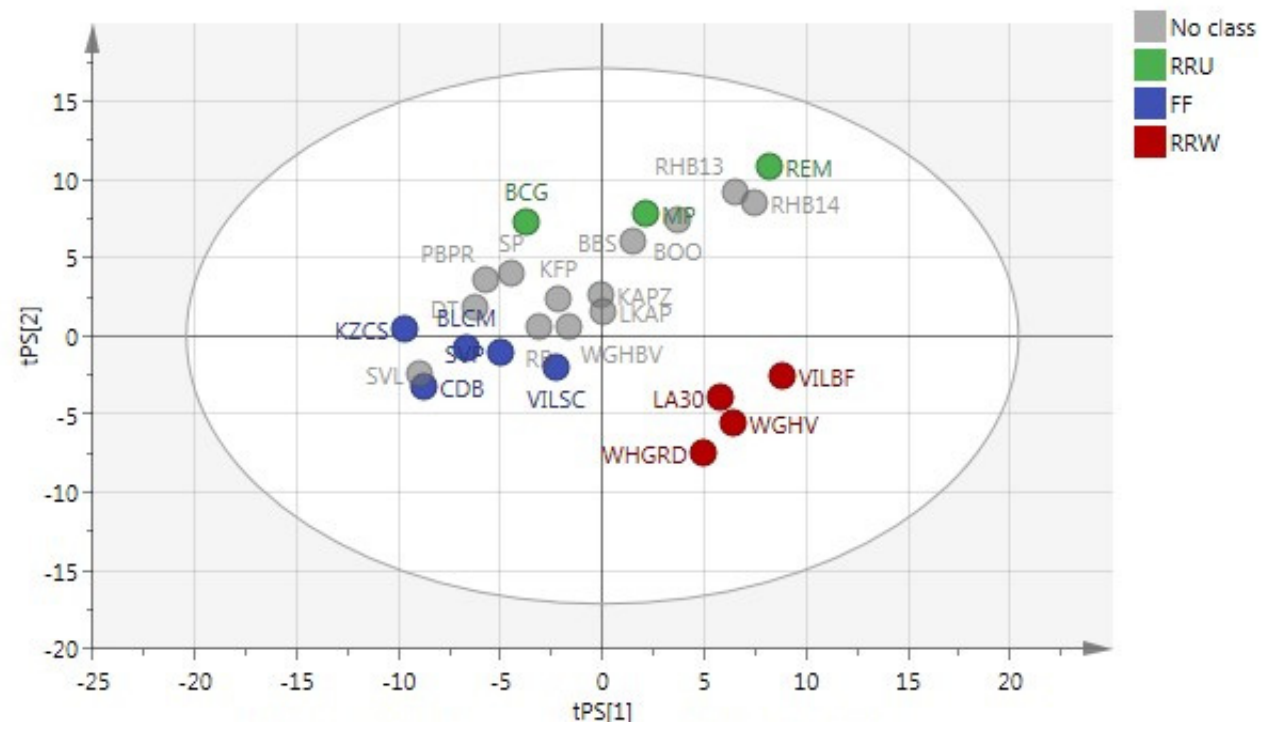

Figure 5. PLS-DA model for the combined LCHRMS results (positive and negative ionization), no sample pre-processing $(n=25)$. Styles attributed by the sensory task. 'No class' samples constitute the prediction set. 
As expected, the DA representation for the samples attributed to styles shows good grouping, which is promising for future work. In order to try and predict to which group the 'no class' samples belong, a PLS-DA model was created, using the wines subjected to sensory analysis as training set. The drawback is in this case the number of samples used to create the model. There were more samples included in the negative ionization mode experiment (39 vs 25), which resulted in a larger prediction set for this MS mode, while the working set remained the same size. A prediction certainty of $50 \%$ resulted in 2 samples being unclassified in positive MS mode ( $n=25), 3$ in negative $(n=39)$, and 1 in combined data sets $(n=25)$. Increasing the level of certainty to $75 \%$ resulted in 7,15 , and 8 unclassified samples, respectively.

The wines that were not attributed clearly to a style group had to be excluded from the training set, too. Interestingly, the chemical analysis places these two sample, LKAP and WGHBV, in the middle of the plot, between the FF and RRU groups (Fig 4 and 5). When looking at the predicted scores for these wines, they fall below $50 \%$ certainty for assigning to a style group. This can possibly explain the difficulty that the judges had in placing these particular samples in either of the styles.

The general configuration is similar when considering the LC-HRMS results from the two ionization modes separately and combined (Fig 4 and 5). Even though the general configurations obtained from separate and combined MS ionization mode data appear similar, the prediction scores change depending on the set used, therefore there is merit in using a more complete set of results. For example, for the samples REM (RRU, training set), and RHB13 and RHB14 (prediction set), their positions change depending on the MS data considered. They are not closely associated in the representation of the positive mode (Fig 4a) but situated together in the representation of the negative and combined data sets (Fig $4 b$ and 5 ). The prediction scores indicate the same. For positive MS mode, RHB13 and RHB14 can be attributed to both RRU and RRW groups with a certainty of around or less than $50 \%$. In negative mode and combined sets, both samples are attributed to the RRU group with a confidence higher than $90 \%$. Some insight into the samples reveals that they are all from the same estate, made in similar conditions, the differences between the two samples in the prediction set is the vintage (therefore, the age at the time of analysis) and that, often, these wines are sensorially characterized as RRU. This particular situation makes the case for combining the ionization mode results to avoid loss of information.

Up to this point, the work presented here can be considered more proof of principle rather than definitive evidence that Chenin Blanc styles can be predicted using LC-MS data. More in-depth data analysis will possibly 
reveal the compounds correlated with styles. The presence of such markers should eliminate the need for insight or sensory information in the case of samples that fall in-between groups. At the same time, removing superfluous MS information would result in decreasing the statistical noise and make predictions more reliable.

\section{CONCLUSIONS}

Results indicate that even though the traditional evaluation of Chenin Blanc styles has its merits, a more objective way of attributing the style is also possible with the help of chemical analysis coupled with integrated statistical tools. Even though models based on chemical data can designate a wine as fitting in a specific group, sensory evaluation has in some cases more relevance, as it deals with human perception, be it for experts or consumers. In the field of sensory research, this translates into a need for a sensory method that can evaluate more wines to increase the training set and create a more reliable model.

The issue of choice for representative samples for the training set could be avoided in the future with the help of marker molecules. Identification of markers for styles would make the discrimination between groups easier, avoiding the issue of wines that fall "in between" the groups in both sensory and chemical evaluation.

\section{EXPERIMENTAL SECTION}

\section{Sensory evaluation: Directed sorting}

A sample set of 15 wines (including 2 repeats) were subjected to sensory evaluation in duplicate by 15 experts using a directed sorting task, taking into account both aroma and taste. The judges were asked to divide the samples into three groups according to the Chenin Blanc wine style. The data has been analysed using DISTATIS to assess individual differences between samples as well as to build a multivariate map of the data using multidimensional scaling (MDS).

\section{Chemical analysis: LC-HRMS}

Wine samples ( $n=39$, including the ones used for sensory evaluation) were analysed by UPLC (Waters Corporation) equipped with a Synapt G2 quadrupole time-of-flight mass spectrometer (Waters Corporation). The 
separation was done on an Acquity UPLC HSS T3 column $(1.8 \mu \mathrm{m}$ internal diameter, $2.1 \mathrm{~mm} \times 100 \mathrm{~mm}$, Waters Corporation) using $0.1 \%$ formic acid (mobile phase A) and acetonitrile (mobile phase $\mathrm{B}$ ) and a scouting gradient. Flow rate was $0.3 \mathrm{~mL} / \mathrm{min}$ and the column temperature $55^{\circ} \mathrm{C}$. The injection volume was $2 \mu \mathrm{L}$.

Data was acquired in $\mathrm{MS}^{\mathrm{e}}$ mode which consisted of a low collision energy scan (6V) from m/z 150 to 600 and a high collision energy scan from $\mathrm{m} / \mathrm{z} 40$ to 600 . The high collision energy scan was done using a collision energy ramp of 30-60 V. The mass spectrometer was optimized for best sensitivity, cone voltage $15 \mathrm{~V}$, nitrogen desolvation gas at $650 \mathrm{~L} / \mathrm{hr}$ and desolvation temperature $275^{\circ} \mathrm{C}$. The instrument was operated with an electrospray ionization probe in both positive and negative mode.

Chromatographic data was extracted as (RT_m/z, intensity) matrix by the application manager used. The MS data generated from both ionization modes (separate and combined sets) was analysed using MarkerLynx XS (Waters Corporation), an application manager that performs 3D peak integration, data set alignment and incorporates multivariate statistical tools. The software is directly integrated with SIMCA-P (Umetrics) and the statistical algorithms are directly applied to the processed data sets.

\section{ACKNOWLEDGMENTS}

The authors would like to thank THRIP and Winetech for the financial support.

\section{REFERENCES}

1. A. De Villiers, P. Majek, F. Lynen, A. Crouch, H. Lauer, P. Sandra, European Food and Research Technology, 2005, 221, 520.

2. A. Tredoux, A. De Villiers, P. Majek, F. Lynen, A. Crouch, P. Sandra, Journal of Agricultural and Food Chemistry, 2008, 56, 4286.

3. L. Jaitz, K. Siegl, R. Eder, G. Rak, L. Abranko, G. Koellensperger, S. Hann, Food Chemistry, 2010, 122, 366.

4. J.L. Aleixandre-Tudo, H. Nieuwoudt, J.L. Aleixandre, W.J. Du Toit, Journal of Agricultural and Food Chemistry, 2015, 53, 1088.

5. O. Tomic, A. Nilsen, M. Martens, T. Næs, LWT - Food Science and Technology, 2007, 40, 262.

6. T. Dahl, O. Tomic, J.P. Wold, T. Næs, Food Quality and Preference, 2008, 19, 103. 
7. O. Tomic, G. Luciano, A. Nilsen, G. Hyldig, K. Lorensen, T. Næs, European Food and Research Technology, 2009, 230, 497.

8. M. Hoffmann, P. Varela, G. Ares, "Novel Techniques in Sensory Characterization Profiling and Consumer", CRC Press, Taylor \& Francis Group, Boca Raton, 2014.

9. D. Cozzolino, W. Cynkar, N. Shah, P. Smith, Analytical and Bioanalytical Chemistry, 2011, 401, 1497.

10. A. Edelmann, J. Diewok, K.C. Schuster, B. Lendl, Journal of Agricultural and Food Chemistry, 2001, 49, 1139.

11. M. Urbano, M.D. Luque De Castro, P.M. Pérez, J. García-Olmo, M.A. Gómez-Nieto, Food Chemistry, 2006, 97, 166.

12. M. Le Moigne, C. Maury, D. Bertrand, F. Jourjon, Food Quality and Preference, 2008, 19, 220.

13. L.M. Schmidtke, A. Rudnitskaya, A.J. Saliba, J.W. Blackman, G.R. Scollary, A.C. Clark, D.N. Rutledge, I. Delgadillo, A. Legin, Journal of Agricultural and Food Chemistry, 2010, 58, 5026.

14. A.L. Fudge, K.L. Wilkinson, R. Ristic, D. Cozzolino, Journal of Agricultural and Food Chemistry, 2012, 60, 52.

15. J.L. Aleixandre-Tudo, I. Alvarez, M.J. Garcia, V. Lizama, J.L. Aleixandre, Czech Journal of Food Science, 2015, 33, 217.

16. CBA. 2016. Chenin Blanc Styles. Available from: http://www.chenin.co.za/styles.html accessed 16 Dec 2016

17. D. Valentin, S. Chollet, M. Lelievre, H. Abdi, International Journal of Food Science and Technology, 2012, 47, 1563. 
